IAC-11-D5.3.3

\title{
COMBINING SOLAR SCIENCE AND ASTEROID SCIENCE WITH THE SPACE WEATHER OBSERVATION NETWORK (SWON)
}

\author{
Volker Maiwald \\ German Aerospace Center (DLR), Institute of Space Systems, Department of System Analysis Space Segment, \\ Bremen, Germany, Volker.Maiwald@DLR.De
}

\begin{abstract}
André Weiß
German Aerospace Center (DLR), Institute of Space Systems, Department of System Analysis Space Segment, Bremen, Germany, Andre.Weiss@DLR.De

Frank Jansen

German Aerospace Center (DLR), Institute of Space Systems, Department of Space Systems, Bremen, Germany, Frank.Jansen@DLR.De
\end{abstract}

The peculiarity of space weather for Earth orbiting satellites, air traffic and power grids on Earth and especially the financial and operational risks posed by damage due to space weather, underline the necessity of space weather observation. The importance of such observations is even more increasing due to the impending solar maximum. In recognition of this importance we propose a mission architecture for solar observation as an alternative to already published mission plans like Solar Probe (NASA) or Solar Orbiter (ESA). Based upon a Concurrent Evaluation session in the Concurrent Engineering Facility of the German Aerospace Center, we suggest using several spacecraft in an observation network. Instead of placing such spacecraft in a solar orbit, we propose landing on several asteroids, which are in opposition to Earth during the course of the mission and thus allow observation of the Sun's far side. Observation of the far side is especially advantageous as it improves the warning time with regard to solar events by about two weeks. Landing on Inner Earth Object (IEO) asteroids for observation of the Sun has several benefits over traditional mission architectures. Exploiting shadowing effects of the asteroids reduces thermal stress on the spacecraft, while it is possible to approach the Sun closer than with an orbiter. The closeness to the Sun improves observation quality and solar power generation, which is intended to be achieved with a solar dynamic system. Furthermore landers can execute experiments and measurements with regard to asteroid science, further increasing the scientific output of such a mission. Placing the spacecraft in a network would also benefit the communication contact times of the network and Earth. Concluding we present a first draft of a spacecraft layout, mission objectives and requirements as well as an initial mission analysis calculation.

\section{INTRODUCTION}

The threat of space weather effects on e.g. Earth satellites, air traffic, power grids or any technical equipment susceptive to electromagnetic radiation is significant. To prevent negative repercussions due to function failures or costly interruptions of services thorough predictions of space weather are mandatory. The importance of these effects or more the prevention of these is recognized in ESA's Space Situational Awareness program and actions of the European Commission [1].

Currently all solar observations used for prediction of solar space weather (e.g. Coronal Mass Ejections (CMEs), Solar Energetic Particle (SEP) events, solar radio bursts and strong changes in X-ray respectively UV emissions of the Sun) are directed at the Earth facing side of the Sun, which means in the best case the maximum practical limit for predictions is several days (the solar rotation period is 28 days at the Sun equator).
Extending observations to the complete solar surface, especially considering the Earth opposing side, could increase the prediction time to 28 days.

While there are several missions planned for solar observations, e.g. Solar Probe (NASA) or Solar Orbiter (ESA), none of them addresses observations on the solar far-side and consequently do not increase prediction times for solar space weather.

Therefore we propose a mission architecture that addresses this problem by placing a network of space weather sensors around the Sun, especially making sure that there are sensors in the viewing area directly behind the Sun.

Under the title Space Weather Observation Network (SWON) it is suggested to use a "fleet" of landing vehicles distributed over Inner Earth Objects (IEOs) for solar monitoring to predict Sun originated space weather with a longer warning time than currently 
achievable, while at the same time use solar dynamic power generation due to the favourable environment.

Using SWON to view at the Earth-opposing side of the Sun could increase the prediction time by a factor of two, or even allow continuous observation of the whole solar "surface".

The scientific usefulness of SWON exceeds solar science and includes asteroid science as well. Landing on IEOs allows in-depth investigation of asteroids, e.g. determination of composition, structure and terrain features.

The idea of such a network has been investigated and discussed in a Concurrent Evaluation session, a method which exploits the infrastructure of DLR's Concurrent Engineering Facility (CEF) for e.g. Mission Architecture Definitions (MAD).

\section{EVALUATION METHOD}

The DLR Institute of Space Systems has a Concurrent Engineering (CE) Facility (CEF) at its disposal, which is used for spacecraft or general system design. At the heart of the Concurrent Engineering process is the idea of assembling the respective subsystem experts and supply the necessary infrastructure to allow direct and quick communication (for problem identification and solving), data exchange (design parameters, e.g. power, mass, dimensions) and visualization of the given system.

So called off-line work is used for calculations, research, data accumulation and documentation, while moderated sessions allow the exchange of data and present the study progress. Arising problems are discussed within the team during these sessions as well.

The CE environment further provides the technical means to distribute data and information, manage the knowledge and consult with study participants which are possibly distant to the study team via video conference.

The usual inputs into a CE-study, as conducted by DLR, are system- and mission requirements, which are formulated before the study starts. To exploit the advantages of the CE environment, Weiß et al. [2] implemented a method for mission evaluation, i.a. for Mission Architecture Definition (MAD).

This approach has been tested with the Space Weather Observation Network (SWON) mission concept in September 2010.

The major questions to be answered during such a micro-study are:

o What are the mission/ science objectives?

o What are the technological challenges (development needs)?

o How and when can the mission target be reached?

o What are the mission risks?

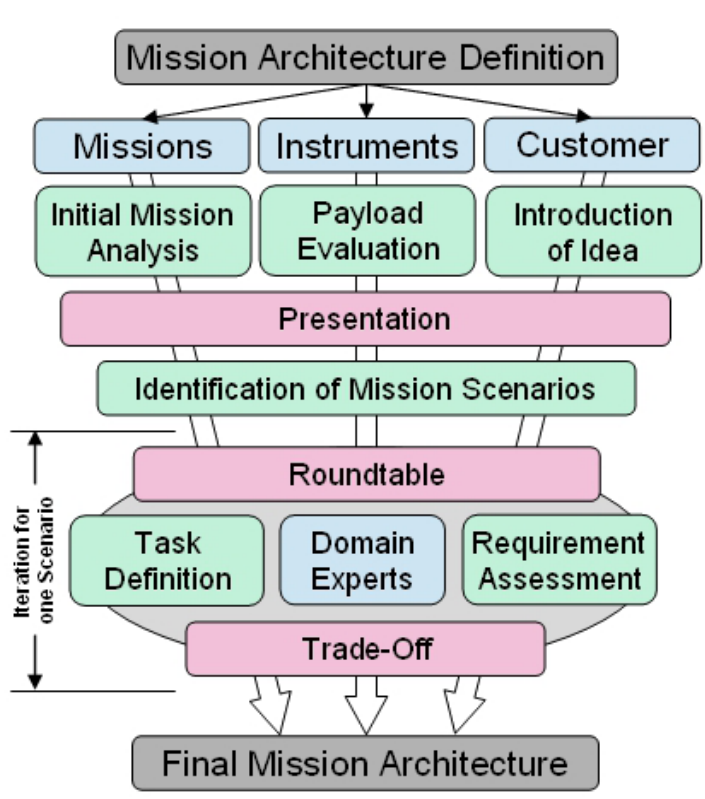

Fig. 1: The Mission Architecture Definition Process as used with Concurrent Evaluation.

The MAD begins with introductory presentations, clarifying on the content of the idea, the science or payload side of the mission and possible mission analysis (Fig. 1), where applicable. Basically, just as with the ordinary CE process, it is ensured that the whole study team has the same minimum level of background information at the start of the session.

Subsequent to these presentations, initial feedback is gathered from the roundtable of experts to identify problems, requirements, general points of discussion.

These discussion points are then weighted to allow the immediate solving of relevant issues in order of their prominence to the mission.

Mission scenarios regarding these discussion points and their importance are then iteratively sketched and evaluated by the design team. Based on the (usually science driven) mission objectives, the design team defines the necessary subsystems study and their tasks as well as the requirements for the system.

Via a trade-off, containing the advantages and disadvantages of each scenario, the final mission scenarios are weighted against each other at the end of the study.

The study outcome, i.e. the final mission architecture, is documented in a report on which future studies, possibly a full-fledged CE-study can be based on. 


\section{MISSION SCIENCE}

The science behind SWON is taken from three fields, solar and asteroid science, as well as technology demonstration.

\section{III.I Science/ Mission Objectives}

The science objectives for the SWON mission can be summarized as follows:

- $\quad$ put the far side of the Sun under observation

- $\quad$ increase the prediction time of solar originated space weather to up to 28 days, and

- technology demonstration of solar-dynamic power generators.

During the course of the study and due to the finally chosen mission architecture, one further objective has been added:

- gather information about the asteroid landing sites, e.g. composition and material strength.

\section{III.II Science Implementation}

Currently only indicators like backscattered Lymanalpha photons can grant information on solar space weather events on the Earth-opposing side of the Sun. To increase the time of reliable predictions of space weather to up to 28 days, it is therefore mandatory to put this region of the solar atmosphere under surveillance.

Two options have been noted by the study team to fulfil that goal:

(1) a telescope based mission could be placed on an orbit of approx. 1 AU solar distance behind the Sun, e.g. at Lagrange Point 3 and

(2) a light-weight mission at closer range utilizing less sophisticated sensors.

Due to the novelty of the scenario as opposed to more traditional solar observation missions, the study team mostly investigated option (2) during the course of the study.

\section{MISSION DESIGN}

To allow a thorough and continuous observation of the solar atmosphere with regard to space weather, it has been determined that a network of several small spacecraft could be used in medium to low solar distance $(<1 \mathrm{AU})$. A mission with the given science objectives should be undertaken during a solar maximum period.

While the demonstration of the concept could take place at another time, the need for a space weather forecast is the greatest during solar maximum. The next solar maximum period is expected for about 2013 to 2015, the following about eleven years later. Therefore the demonstrator mission could be implemented during the former, the full-fledged network during the latter timeframe.

\section{IV.I System Design}

It has been discussed and found as a valid option by the study team to use existing Inner Earth Objects (IEOs) as basis for the intended observations by placing a sensor suit on some of them, thus creating an observational network for the Sun by a group of landers.

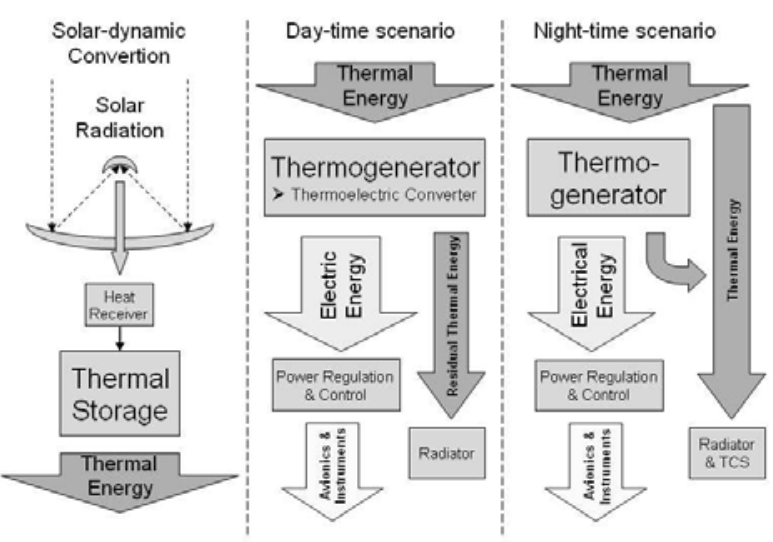

Fig. 2: Concept of the intended solar-dynamic system for an asteroid mission: Thermal energy is used as an alternative to solar cells and batteries and to heat the spacecraft during eclipse periods.

\section{IV.II Power Generation and Asteroid Environment}

Solar-dynamic (SD) systems have been in discussion as space based power source and have also been tested on Earth in the timeframe from 1992 to 1998, however no mission has yet been undertaken with this technology [3].

Due to the rotational period of a few hours, asteroids present a suitable environment for SD systems. After being charged during illuminated periods close to the Sun, the heat provided by the thermal storage can be used to generate power during the eclipse periods (cf. Fig. 2).

For photovoltaic systems it is common to reduce or cease scientific operations at eclipse times as the thermal subsystem requires an increased amount of power to convert electrical to thermal energy and keep the spacecraft on a sufficiently high temperature. Spacecraft designs for the exploration of the inner solar system usually implement additional thermal insulation and radiators with respect to the higher solar radiation density. By landing on an asteroid, the lander gains cool-down time due to rotating out of illumination.

While the asteroids' rotation is beneficial from a thermal point of view, it precludes the primary mission objective of continuous solar observation. This can be 
remedied by a second lander positioned on the opposite asteroid side.

Investigating the orbits of the possible IEO targets (s. Tab. 1 for a list), no asteroid is always opposing Earth, i.e. several landers are required to ensure full coverage of the Earth-opposing side of the Sun.

\begin{tabular}{|c|c|c|c|c|}
\hline & Period[y] & $\mathrm{a}$ [AU] & e & $\mathrm{i}\left[{ }^{\circ}\right]$ \\
\hline $2003 \mathrm{CP}_{20}$ & 0.637 & 0.741 & 0.322 & 25.617 \\
\hline $2004 X_{130}$ & 0.485 & 0.617 & 0.454 & 2.953 \\
\hline $1996 \mathrm{DK}_{36}$ & 0.576 & 0.692 & 0.415 & 2.017 \\
\hline $2004 \mathrm{JG}_{6}$ & 0.506 & 0.635 & 0.531 & 18.945 \\
\hline $2005 \mathrm{TG}_{45}$ & 0.562 & 0.681 & 0.372 & 23.329 \\
\hline 2006 KZ39 & 0.475 & 0.609 & 0.541 & 9.925 \\
\hline 2006 WE4 & 0.695 & 0.784 & 0.182 & 24.767 \\
\hline 2007 EB26 & 0.405 & 0.548 & 0.786 & 8.461 \\
\hline 2008 EA32 & 0.483 & 0.615 & 0.304 & 28.262 \\
\hline 2008 UL90 & 0.579 & 0.694 & 0.380 & 24.307 \\
\hline
\end{tabular}

Table 1: Properties (orbit period, semimajor axis, eccentricity and inclination) of Inner Earth Objects known during the SWON study.

These ten IEOs have been grouped with respect to similar inclinations with the biggest and therefore most promising group consisting of four asteroids with an inclination from 23.329 up to 28.262 degrees (while a large inclination results in large Delta- $\mathrm{V}$ requirements, it would also benefit observation of solar polar regions). The next step was to determine whether this subset of asteroids could be utilized to realize the full network scenario. By accessing the JPL HORIZONS database system, the ephemerides for the solar maximum timeframe 2024 until 2026 have been identified. This allowed calculating the angle between each asteroid and Earth (in the solar system barycenter reference system).

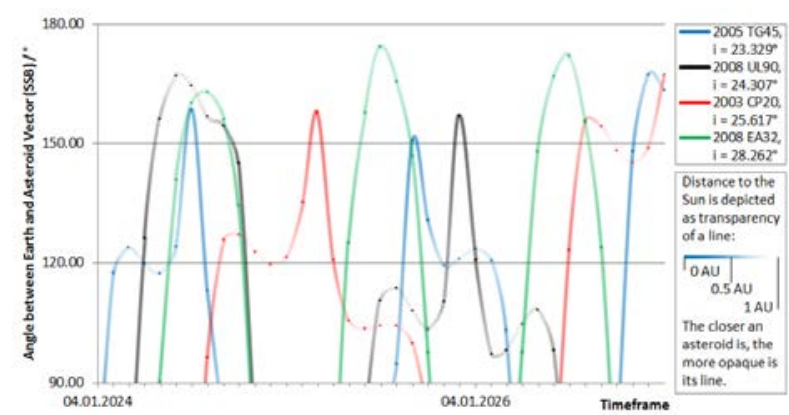

Fig. 3: Earth opposing Inner Earth Objects with an inclination from 23.329 up to $28.262^{\circ}$. Displayed are angles between Earth and each asteroid as a function of time during a period of expected solar maximum with a resolution of one data-point per month.

Fig. 3 displays angles greater than $90^{\circ}$ for the group of four asteroids. The most promising interval with all asteroids consecutively passing the Sun directly opposite Earth lasts from February 2025 until January 2026: With only one exception (2008 UL90, July 2025, 0.76 Astronomical Units distance to the Sun) we can find at least one asteroid with a distance of $0.66 \mathrm{AU}$ or less covering the far side of the Sun.

Through the transparency of a line, Fig. 3 also signifies the distance of an asteroid to the Sun. The darker depiction of asteroids 2003 CP20, 2005 TG45 and 2008 UL90 during their respective maxima in the before highlighted interval explains, why the actual point of opposition (i.e. an angle of $180^{\circ}$ ) is not depicted: During these closer approaches and the tantamount higher velocity (third law of Kepler), the chosen resolution of the ephemerides is not sufficient and the $180^{\circ}$ "swept" within less than a month. Still, the curve maxima denote, when it is to be expected. Asteroid $2008 \mathrm{EA}_{32}$, which passes the point of opposition further away from the Sun (higher line transparency during the maximum), is slower: Here, the resolution becomes more appropriate, depicting the maximum at about $174^{\circ}$.

It can be summarized that the chosen four asteroids present a good opportunity to realize the envisioned network scenario to cover the solar far side throughout the identified interval.

The environment of an asteroid was identified as problematic for the mission. First, the rotation periods and axes of asteroids are usually not stable, which means predictions about observation durations, target acquisition of the Sun, etc. are difficult to make. Data about the rotational period of the intended target group (IEOs) is currently scarce but can be obtained when necessary.

Actual landing on the target can be complicated due to uneven surface structures and generally unknown properties like e.g. surface strength. Low gravity of the target requires anchoring on the surface.

As for the actual network more than one target body is necessary, it has to be checked how dissimilar the possible targets are regarding their properties and if it is possible to design a lander suitable for a broad range of asteroids or if each lander has to be individually adapted to its target.

It also has to be investigated whether the heated rock/ material of the asteroid around the lander has effects on the thermal generator process or whether it can even be exploited.

During the first discussion round, the study team determined that the available scientific data of IEOs and of asteroids in general is not sufficient to enable a reliable design of the necessary network. Of the listed asteroids, only Atira $\left(2003 \mathrm{CP}_{20}\right)$ has a known rotational period (2.9745 hours). Furthermore information about the composition and the surface properties of these asteroids is presently also not available. 
Considering this, the network solution was postponed and a preceding demonstrator was proposed by the study team.

This mission will consist of a single lander with two tasks: to realize the lightweight option described before and to improve knowledge about asteroids with dedicated instruments. The latter will amount for $50 \%$ of the payload and will be based on the Mobile Asteroid Surface Scout (MASCOT) concept of DLR [4].

While a full SD system has not been realized, the required technologies can already be found in current spacecraft or rovers. Politically and socially criticaleyed RTGs yield methods to convert thermal to electrical power. The critical element - the nuclear source - can hereby be replaced through solar heat. The basic element of common RTGs are thermocouples. Thermocouples are based on the Seebeck effect and generate voltage when applied with a thermal gradient. They commonly have efficiencies between 3 and $7 \%$.

While already in use, e.g. on the Cassini spacecraft, the rather low efficiency rate lead to the development of a more effective heat converter, intended for future deep space missions. It is called "Advanced Stirling Converter" and can achieve efficiency rates of up to $30 \%$ [7].

Current models reach a mass specific power of $3.5 \mathrm{~W} / \mathrm{kg}$ at $850^{\circ} \mathrm{C}$ and the smallest available unit has a mass of $1.5 \mathrm{~kg}$, resulting in an available output of $5.25 \mathrm{~W}$. Initial calculation results in a concentrator area of $1.09 \mathrm{~m}^{2}$

\section{IV.III Preliminary Payload}

Due to the small solar distance $(<1 \mathrm{AU})$ a sensor suit without a telescope suffices to fulfill the observational mission objectives of the Sun.

- $\quad$ Flux-Gate Magnetometer

- $\quad$ Particle Monitor

- Gamma- and X-Ray Flux Monitor

- $\quad$ EUV Flux Monitor

- $\quad$ Small Optical Camera

The overall mass estimation for all listed instruments is $1.5 \mathrm{~kg}$ while the power budget is about $2.5 \mathrm{~W}[5,6]$. Measurements shall be taken at a frequency of $1 \mathrm{~Hz}$ and should be transmitted to Earth every 60 minutes to allow a high data density.

For the asteroid science an additional payload of 6.1 $\mathrm{kg}$ is considered based on the instruments of MASCOT [4]:

- APXS, the A-Particle X-ray Spectrograph to analyze the chemical surface composition,

- $\quad$ Neo-Mole/HP3, a soil sampling device capable of going below the surface,
- $\quad$ ILMA, the ion laser mass analyzer for solid and volatile samples on molecular and isotopic scale and

- MicrOmega, an optical microscope and IR spectrometer to characterize soil samples at grain scale $(<10 \mu \mathrm{m})$ with regard to size, distribution, morphology and mineralogy

The total maximum mass for the landers has been set to $43 \mathrm{~kg}$, based on MASCOT.

\section{IV.IV System and Mission Requirements}

Tables 2 and 3 list the preliminary mission and system requirements as formulated by the study team.

\begin{tabular}{|c|c|c|}
\hline & Demonstrator & Network \\
\hline $\begin{array}{l}\text { Mission } \\
\text { Duration }\end{array}$ & $\begin{array}{l}2 \text { years to cover } \\
\text { the complete solar } \\
\text { maximum }\end{array}$ & $\begin{array}{l}>2 \text { years, to justify } \\
\text { costs }\end{array}$ \\
\hline $\begin{array}{l}\text { Target } \\
\text { Acquisition }\end{array}$ & $\begin{array}{l}\text { Minimum of } 14 \\
\text { days observation } \\
\text { time (half a } \\
\text { rotation) }\end{array}$ & $\begin{array}{l}\text { Continuous } \\
\text { observation }\end{array}$ \\
\hline
\end{tabular}

Misc.

Asteroid composition and properties need to be known

Table 2: Preliminary mission requirements for SWON.

\begin{tabular}{lll}
\hline Max dry mass & Demonstrator & Network \\
\hline Instruments & $\begin{array}{l}\text { 900 kg (scaled up } \\
\text { from demonstrator } \\
\text { for several landers) }\end{array}$ \\
Payload mass & $\begin{array}{l}\text { Equal share of instruments for solar and } \\
\text { asteroid science (ca. 5-6 kg total) }\end{array}$ \\
Communication & Undisrupted contact to Earth \\
\hline Table 3: Preliminary system requirements for SWON.
\end{tabular}

\section{MISSION ANALYSIS}

Before the study a preliminary mission analysis has been conducted to investigate the feasibility of the target area and bodies. After the study with the new input a more thorough investigation has been conducted.

As can be seen in Table 1, the targets have perihelions close to the Sun, usually very eccentric orbits $(\mathrm{e}>0.3)$ and some have very high inclinations (i $>20^{\circ}$ ). Overall the target group has varying orbit properties and cannot be considered a family. In general this results in large Delta- $\mathrm{V}$ requirements for the mission. To address these, the initial mission analysis has been processed under the assumption of utilizing solar electric propulsion. 
Two different approaches were investigated:

- $\quad$ attempting to rendezvous with as many targets as possible and

- $\quad$ attempting to flyby as many targets as possible, assuming the actual rendezvous is conducted by the lander vehicle itself

\section{V.I Calculation Method}

As the mission was from the beginning assumed to be of solar electric propulsion nature, the code InTrance [9], has been used throughout the mission analysis.

This code utilizes a combination of evolutionary low-thrust trajectories. The optimization goal has in this case always been minimization of fuel consumption as fuel savings can be used for an additional rendezvous.

The launch dates have been one hundred days onwards from $18^{\text {th }}$ April and one hundred days from $31^{\text {st }}$ March 2024 onwards. The launch dates have been chosen with regard to possible development times and the needs due to solar maxima.

A rendezvous has been considered for a difference in heliocentric velocities of $200 \mathrm{~m} / \mathrm{s}$.

\section{V.II Calculation Models}

For the course of the mission analysis calculations

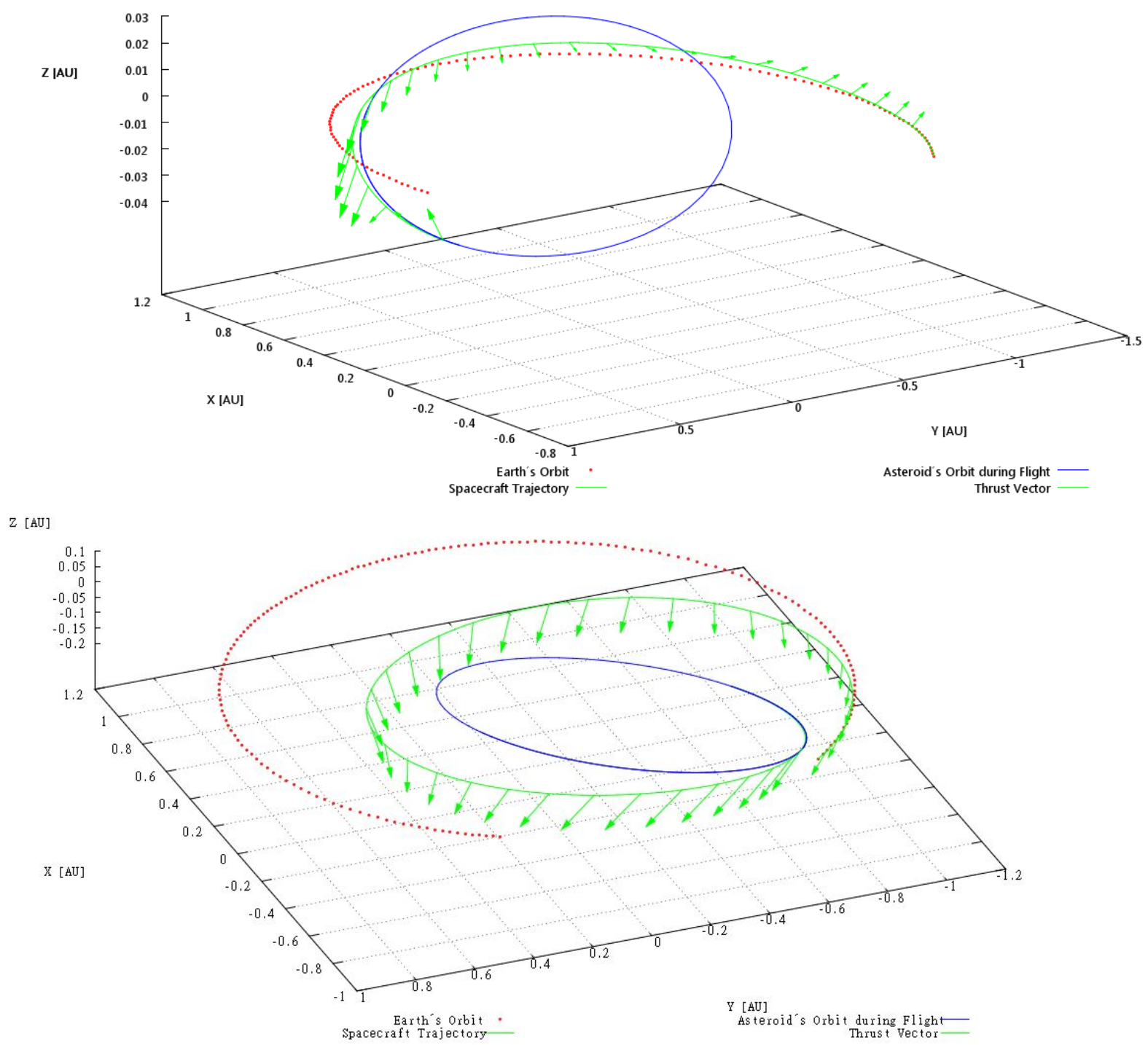

Fig. 4: Post-study rendezvous trajectories targeting $2006 \mathrm{KZ}_{39}$ (top) and $2004 \mathrm{XZ}_{130}$ (bottom) The outer, dotted line represents Earth's orbit, while the most inner, continuous line maps the asteroid's orbit. The spacecraft trajectory can be distinguished through the supplemental vectors, depicting the thrust direction

algorithms and artificial neural networks to optimize the following models were applied: 
Spacecraft

The spacecraft parameters used for the mission analysis calculations have been:

- $\quad$ Dry mass: $250 \mathrm{~kg}$

- $\quad$ Solar power at $1 \mathrm{AU}: 14 \mathrm{~kW}$

- $\quad$ Main engine: 2 RIT-22 ME thrusters (60 kg)

- $\quad$ Specific impulse $\mathrm{I}_{\mathrm{sp}}=4700 \mathrm{~s}$

\section{$\underline{\text { Solar System }}$}

For the calculations the central body has been the Sun. The launch body has been Earth, the IEOs listed in Tab. 1 have been the group of possible target bodies. For perturbations Venus and Mercury have been considered due to their closeness to the target area.

\section{V.III Calculation Results}

For a launch date in May 2013 a possible target opportunity is found with an arrival at $2006 \mathrm{KZ}_{39}$ in March 2014. The fuel mass ratio for this rendezvous is 0.44. The rendezvous trajectory is depicted in Fig. 4, top. It can be seen that the spacecraft spirals down from Earth to the target asteroid. Final velocity difference to the target is $188.2 \mathrm{~m} / \mathrm{s}$ and the accuracy of the trajectory is $21,490 \mathrm{~km}$ (limitations have been $200 \mathrm{~m} / \mathrm{s}$ and $100,000 \mathrm{~km})$.

The rendezvous takes place behind the Sun, viewed from Earth. As a launch date of 2013 is not realistic given the development effort that currently still needs to be addressed, a second launch window for the solar maximum in 2025 has been investigated. The optimization provided a rendezvous with $2004 \mathrm{XZ}_{130}$ for late December 2024, after a flight time of ca. half a year. This target is reachable with a fuel mass ratio of 0.23 ; the trajectory is depicted in Figure 4, bottom. The velocity difference is $0.108 \mathrm{~m} / \mathrm{s}$ and the accuracy 693.2 $\mathrm{km}$.

It can be seen that the trajectory reaches its target closer to Earth, resulting in less fuel consumed and a shorter flight time, comparing to the previous trajectory.

However the rendezvous takes place in front of the Sun, when viewed from Earth.

For further in-depth mission analysis, first of all the targets need to be chosen more carefully regarding their relative position to the Sun in the desired timeframes. It could also be investigated if Near Earth Objects that cross Earth's orbit could be suitable as targets for deploying landers if they carry the lander closer to the Sun than Earth's solar distance and would place them behind the Sun for solar observation.

\section{OPEN ISSUES}

As the SWON concept has been in investigated in a MAD of one day (and intensive preparation and analysis in the aftermath), there are several issues that need further in-depth attention as they are mission critical. These issues include the investigation of:

\section{Communication Concept}

- How can a durable communication link with Earth be ensured?

- Data transfer has to be allowed at least once every hour.

- Is the network architecture exploitable for communications?

- $\quad$ Can the carrier vehicle be used as a relay?

\section{Science on the carrier vehicle}

- Can the carrier vehicle of the landers be used for further science, if so, how?

- What scientific payload should be onboard the demonstrator and full network option besides the lander vehicles?

\section{Solar Dynamic Power Generator}

- Operational characteristics need to be specified

- What effects are caused by vibrations?

- What medium is used for heat storage and what are its properties?

\section{Target rendezvous scheme}

- Should the rendezvous with each target be conducted by the carrier vehicle or by each lander itself after a flyby of the carrier vehicle?

\section{CONCLUSION}

It has been shown how a network of asteroid landers, placed on Inner Earth Objects could help with the prediction of solar originated space weather effects by extending the observation time to up to 28 days. The mission analysis supports the claim of feasibility whereas none of the requirements seem to be far-fetched for current technological standards. We therefore propose the Space Weather Observation Network as a means to provide continuous surveillance data of the solar atmosphere.

\section{ACKNOWLEDGEMENT}

The authors thank the whole SWON study team for their enthusiastic and creative participation in the study.

\section{REFERENCES}

Documents ESA Ministerial Council, 25-26 November 2008, see in ESA PR-44, Den Haag, 2008 
[2] Weiß, A., Maiwald, V., Wübbels, G., Concurrent Evaluation - An Application for DLR's Concurrent Engineering Facility, SECESA 2010, 13-15 ${ }^{\text {th }}$ October 2010

[3] Shaltens, R.S., Mason, L.S., 800 Hours of Operational Experience from a 2 kWe Solar Dynamic System, NASA/ TM -1999-208840, 1999

[4] Lange, C., Dietze, C., Ho, T.-M., et al. Baseline Design of a Mobile Asteroid Surface Scout (MASCOT) for the Hayabusa-2 mission, IPPW-7, 2010, Barcelona, Spain

[5] Jansen, F., Behrens, J., Pospisil, S., Kudela, K., Space situational awareness satellites and ground based radiation counting and imaging detector technology, Nuclear Instruments and Methods in Physics Research A633, S231S234, 2011

[6] Jansen, F, Behrens J., Cosmic Rays for Heliospheric Space Weather Storm Prediction, Proc. $12^{\text {th }}$ ICATPP Conf. Como, eds. S. Giani, C. Leroy, P.G.Rancoita, ISBN 978-981-432902-6, 2011

[7] Wong, W.A., Wood, J.G., Wilson, K., Advanced Stirling Convertor (ASC) - From Technology Development to Future Flight Product, Glenn Research Center, 2008

[8] Dachwald, B., Low-Thrust Trajectory Optimization and Interplanetary Mission Analysis Using Evolutionary Neurocontrol, Doctoral Thesis, University of the Federal Armed Forces Munich, Germany, 2004 